stood Mendelian inheritance well. They found it virtually impossible to accept the concept of the healthy carrier. The usual response was to agree politely that the child was perfectly healthy and did not need any treatment, but that children should be tested as young as possible- "so that it can be treated in time."

Since completing this study we have been aware of the comparative simplicity of counselling families who come for testing, or have already been tested, if there is prior knowledge of their condition. They might, for example, have relatives with a haemoglobinopathy or trait, might have been tested themselves in pregnancy, or might have been referred by a doctor of their own ethnic background.

We conclude that mass screening for haemoglobinopathy is unjustified, at least in this country. We advocate instead further health education about haemoglobinopathies together with financial support for hospitals recognised as able to offer both adequate testing and counselling. The UK Thalassaemia Society's booklet contains a list of such hospitals in the London area.

Department of Paediatrics

MARy A Rossiter MARIA SOLOMON

Department of Haematology, London N18 1QX

Department of Haematology, Prince of Wales's Hospital, London N15

G W MARSH

JEANNE D REEve

Catherine S Peckham

Department of Community Medicine,
Charing Cross Hospital, London W6 8RF

\section{Vaginal bleeding in early pregnancy}

SIR,-At the end of your leading article "Mortality in the British rubber industries 1966-76" (16 August, p 471), you remark, "At a time of financial stringency money should be spent on preventive measures only when the evidence is sound and not simply because doubts have been raised." It is a pity that in your leading article "Vaginal bleeding in early pregnancy" ( $p 470$ ) you did not think about that warning before advocating hospital antenatal care, serial oestrogen estimations, ultrasonography, and cardiotocography for every woman who bleeds in early pregnancy.

You rather reluctantly concede that some of this bleeding may be "physiological." In these women the extremely expensive surveillance suggested is not only unnecessary but also meddlesome. You might usefully have suggested ways in which we general practitioners could differentiate between those women in whom bleeding compromises the fetus and those in whom it does not, instead of making a recommendation which (if we acted on it) would further overload hospital antenatal clinics.

Those who preach and practise this sort of defensive obstetrics might find it interesting to join the end of the queue at their next antenatal clinic and find out for themselves what it feels like to be processed in the modern manner. We all have the safety of the mother and child at heart, but surely we should have very sound reasons indeed, certainly sounder than those your editorial advances, for asking a woman to submit to the delays, anxieties, and indignities of high-technology hospital antenatal care.

It is also an awful waste of money. The general practitioner is paid $£ 37.90$ for a woman's antenatal care. I dread to think what the corresponding hospital costs would be.

Health Centre,

J C Bignall

Newport, Dyfed SA42 0TJ

SIR,-Your leading article (16 August, p 470) omits to mention that there are infective causes of bleeding in pregnancy, notably Trichomonas vaginalis infection and gonorrhoea. At the speculum examination vaginal and cervical swabs may well reveal an avoidable cause of "threatened abortion."

Marlborough Clinic,

Royal Free Hospital,

D C MACDONALD BURNS

\section{Screening for the small-for-dates fetus}

SIR,-I note with great interest the comment on my letter made by $\mathrm{Dr} J \mathrm{P}$ Neilson and others (12 July, p 147). I suspect that both sides are partially correct. If we use crownrump length $\times$ trunk area (CRLA) we identify correctly $94 \%$ of small-for-dates fetuses, and with them a number of normal ones which depends on the prevalence of small-for-dates fetuses in the population under test. Using crown-rump length $\times$ trunk circumference (CRLC) we identify correctly $89 \%$ of smallfor-dates fetuses and a rather smaller number of normals. If we ignore the test altogether, simply treating all fetuses as small-for-dates, we identify correctly $100 \%$ of small-for-dates fetuses and a very large number of normals. Which of these is the optimal policy depends on the relative risks of allowing small-for-dates fetuses to go to term and premature delivery of normal ones.

Space does not permit me to deal with the problems of applying monitoring to identify the false-positives generated at the first stage of testing. Instead I wish to submit a mathematical model relevant to the situation over which we are arguing for your readers' scrutiny and criticism.

\section{Definition of terms}

$k$ is the proportion of the tested population with the disease (prevalence);

$T$ is a general test with only two outcomespositive and negative;

$S$ is the sensitivity of $\mathrm{T}$, defined as the proportion of a population consisting entirely of positives that $T$ will identify as such;

$s$ is the specificity of $T$, defined as the proportion of a population consisting entirely of negatives that $T$ will identify as such;

$r$ is the probability of a morbid event occurring in a subgroup of the main population which is defined by the suffix of $r$;

$D$ is the total number of morbid events occurring in a population of unit size.

\section{Suffixes}

1 and 2 are suffixes of $\mathrm{T}, S, s$, and $D$ referring to two tests. The suffixes defining the subgroups to which risks $r$ pertain are:

dis, $R x$ The group which has the disease and to which treatment is given.

,$- R x$ The group which does not have the disease, but to which treatment is given.

dis,- The group which has the disease, but to which no treatment is given.

-,- The group which does not have the disease and to which no treatment is given.

If $S_{1}>S_{2}$ and $s_{1}>s_{2}, T_{1}$ is a better criterion of management under all circumstances than is $T_{2}$. The problem to which this model addresses itself is the situation in which $S_{1}>S_{2}$ but $s_{1}<s_{2}$. The object of management on the basis of $T_{1}$ and $T_{2}$ is to minimise $D$. We shall investigate under what circumstances $T_{2}$ is a better criterion of management than $T_{1}$, even though it is less sensitive. We can identify four groups, and their sizes and their associated risks for the general test $T$.

$\begin{array}{lll}\text { Group } & \text { Size } & \text { Associated risk } \\ \text { True }+ & k S & r_{\mathrm{d} 1 \mathrm{~s}, \mathrm{Rx}} \\ \text { False- } & k(1-S) & r_{\mathrm{d} 1 \mathrm{~s},-} \\ \text { True - } & (1-k) s & r_{-}- \\ \text {False + } & (1-k)(1-s) & r_{-, \mathrm{Rx}}\end{array}$

Thus $D=k S r_{\mathrm{d} 18, \mathrm{Rx}}+k(1-S) r_{\mathrm{d} 1 \mathrm{~s},-}+(1-\mathrm{k}) s r_{-{ }_{-}}+$ $(1-k)(1-s) r_{-}, \mathbf{R x}$. Writing two such equations for $T_{1}$ and $T_{2}$ respectively, subtracting and simplifying we obtain $D_{1}-D_{2}=k\left(S_{1}-S_{2}\right)\left(r_{\mathrm{d} 1 \mathrm{~s}}, \mathrm{Rx}-r_{\mathrm{d} 1 \mathrm{~s}},-\right)+(1-k)\left(s_{1}-S_{2}\right)$

$$
\left(r_{-},-r_{-}, \mathrm{Rx}\right) \text {. }
$$

For $\mathrm{T}_{2}$ to be a better criterion of management than $\mathrm{T}_{1}, D_{1}-D_{2}>0$.

Making this substitution in (2) and simplifying we obtain

$\left.\left((1-k)\left(s_{2}-s_{1}\right)\right) / k\left(S_{1}-S_{2}\right)\right)>\left(\left(r_{\mathrm{d} 18,-}-r_{\mathrm{d} 1 \mathrm{~s}, \mathrm{Rx}}\right) /\right.$ $\left.\left(r_{-}, \mathbf{R x}^{-} r_{-},-\right)\right)$,

provided $r_{-}, \mathbf{R x}>r_{-}, \ldots$. The inequality is reversed if $T_{1}$ is a better parameter than $T_{2}$. For simplicity, we shall define the right-hand side of (3) as a risk ratio, $R$. Rearranging to make $k$ the subject yields

$$
k<\frac{\left.\left(s_{2}-s_{1}\right) / S_{1}-S_{2}\right)}{R+\left(s_{2}-s_{1}\right) /\left(S_{1}-S_{2}\right)}
$$

It is thus possible to determine the prevalence below which greater specificity is at a premium over greater sensitivity.

Applying (4) to the parameters CRLA $\left(\mathrm{T}_{1}\right)$ and CRLC $\left(T_{2}\right)$, we find that $\left(s_{2}-s_{1}\right) /\left(S_{1}-S_{2}\right)=$ $0 \cdot 6$. As workers in the relevant field, $\mathrm{Dr}$ Neilson and his colleagues are better placed than I to estimate $R$, and thus to determine whether CRLA or CRLC is the better criterion of management at the prevalence of small-for-dates fetuses in their experience.

\section{R H KLIPSTEIN}

Neurology Department,

London N195NF

Fetal subdural haemorrhages presenting as hydrocephalus

SIR,-In their recent article Dr M J Robinson and others (5 July, p 35) suggest that hydrocephalus may result from subdural haemorrhage in utero secondary to maternal anticoagulation with warfarin. We are concerned about the lack of confirmatory information for the diagnosis of hydrocephalus. In addition, recent data from this institution prompts us to caution against the assignment of specific factors in the development of intracranial haemorrhage and hydrocephalus.

The determination of hydrocephalus was apparently made on fetal biparietal diameter alone. There was no mention of other ultrasound parameters which are required for this determination, nor were confirmatory necropsy findings reported. The diagnosis of fetal and neonatal hydrocephalus requires the evaluation of the ventricular:cortical ratios for the lateral ventricular system. An abnormally enlarged biparietal diameter is not diagnostic of hydrocephalus. In this case the biparietal diameter of $10 \cdot 4$ may have been the result of the proposed intrauterine subdural haematoma or several other possible factors.

We have been impressed with the advantages 